\title{
Vertical Transmission in a Two-Strain Model of Dengue Fever
}

\author{
David Murillo ${ }^{1}$, Susan A. Holechek ${ }^{1,2,}{ }^{*}$, Anarina L. Murillo ${ }^{1}$, \\ Fabio Sanchez ${ }^{1,3}$, Carlos Castillo-Chavez ${ }^{1}$
}

\begin{abstract}
The role of vertical transmission in vectors has rarely been addressed in the study of dengue dynamics and control, in part because it was not considered a critical population-level factor. In this paper, we apply the pioneering modeling ideas of Ross and MacDonald, motivated by the context of the 2000-2001 dengue outbreak in Peru, to assess the dynamics of multi-strain competition. An invading strain of dengue virus (DENV-2) from Asia rapidly circulated into Peru eventually displacing DENV-2 American. A host-dengue model that considers the competing dynamics of these two DENV-2 genotypes, the resident or the American type and the invasive more virulent Asian strain, is introduced and analyzed. The model incorporates vertical transmission by DENV-2 Asian a potentially advantageous trait. Conditions for competitive exclusion of dengue strains are established. The model is used to show that lower transmission rates of DENV-2 Asian are sufficient for displacing DENV-2 American in the presence of vertical transmission.
\end{abstract}

Keywords: vector-host model, dengue, epidemiology, vertical transmission, Peru

\section{Introduction}

Sir Ronald Ross (1911) first introduced mathematical models in the study of vector-borne disease dynamics, in the context of Malaria [11,66]. Over a decade later Kermark and McKendrick [41] adapted his work in the context of communicable diseases. Today a simplified version of the Kermark McKendrick model is found in most elementary calculus textbooks and is known as the Susceptible-Infected-Recovered or SIR model (Figure 1). The SIR

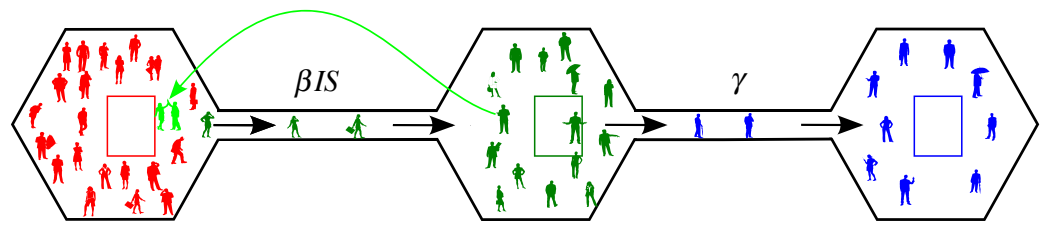

Figure 1: Simple SIR model. Susceptible, $S$, infected $I$, and recovered, $R$, individuals. Parameter $\beta$ represents the per capita per infective rate and $\gamma$ denotes the per capita recovery rate.

\footnotetext{
${ }^{1}$ Simon A. Levin Mathematical, Computational, and Modeling Sciences Center, Arizona State University

${ }^{2}$ Center for Infectious Diseases, Biodesign Institute, Arizona State University

${ }^{3}$ Escuela de Matematica, Universidad de Costa Rica, San Jose, Costa Rica

${ }^{*}$ Correspondence: Susan.Holechek@asu.edu
} 
model is given by the following system of nonlinear differential equations:

$$
\frac{d S}{d t}=-\beta \frac{S I}{N}, \quad \frac{d I}{d t}=\beta \frac{S I}{N}-\gamma I, \quad \frac{d R}{d t}=\gamma I .
$$

The basic reproductive number $\left(\mathcal{R}_{0}\right)$ for System (1) is a dimensionless ratio, $\mathcal{R}_{0}=\beta / \gamma$. The product of the transmission per person rate per unit of time $(\beta)$ and the average infectious period $(1 / \gamma), \mathcal{R}_{0}$ determines whether or not an outbreak will take place depending on whether or not $\mathcal{R}_{0}>1$ or $\mathcal{R}_{0}<1$. If $\mathcal{R}_{0}>1$, then the number of secondary infections generated by a single infectious individual in a pool of purely susceptible individuals (proportion of susceptible in the total population is 1) over his/her average infectious period will be enough for an epidemic outbreak. On the other hand, if $\mathcal{R}_{0}<1$ then the number of secondary cases generated by a typical infectious individual over his/her mean infectious period will not be enough to sustain growth in the class of infectious individuals and the disease will die out. It is also possible to compute the final epidemic size relationship whenever $\mathcal{R}_{0}>1$. For a rescaled system we have that

$$
\ln \frac{s_{0}}{s_{\infty}}=\mathcal{R}_{0}\left(1-s_{\infty}\right)
$$

where $s_{0}$ is the proportion of susceptibles at time $t=0$ and $s_{\infty}$ is the proportion of susceptibles left at the end of the outbreak, that is, at time $t=\infty$. Since the proportion of infectives (at $t=\infty$ ) is equal to zero then by testing for the presence or absence of antibodies to the disease (responsible for the outbreak) prior and after the outbreak, we can estimate $\mathcal{R}_{0}$ from this last relation (for extensions see [35]). The model developed by Ross (1911), now typically referred to as the Ross-MacDonald model, was the first to consider the transmission of a disease that involves a vector and a host. Ross and MacDonald identified via their model the importance of, for example, the host vector ratio, biting rates, and host and vector average infectious periods via the corresponding $\mathcal{R}_{0}$ to the dynamics of malaria. They used their $\mathcal{R}_{0}$ to identify and evaluate control efforts for reducing or eliminating malaria within a host population.

This manuscript provides a detailed application of an extension of the Ross-MacDonald model in the context of a disease, dengue, that is creating havoc in Southeast Asia and the Americas. Dengue, a re-emerging vector-borne infectious disease, puts $40 \%$ of the global population at risk with 50 to 100 million infections per year, including 500,000 cases of dengue hemorrhagic fever (DHF) and 22,000 deaths, mostly in children [17, 47]. The challenges posed by dengue in the U.S. have attracted increased attention due to global mobility [57]. Dengue virus belongs to the genus Flavivirus, family Flaviviridae with four active antigenically distinct serotypes, DENV-1, DENV-2, DENV-3, and DENV-4, [23] and a new DENV-5 serotype, just identified [62]. The pathogenicity of the disease ranges from asymptomatic, mild dengue fever (DF), to dengue hemorrhagic fever (DHF), and dengue shock syndrome (DSS), with children remaining the most affected [32, 60]. DF is characterized by a high fever for 3-14 days with possible symptoms including frontal headache, retro-orbital pain, hemorrhagic manifestations, and rash [16]. A more severe and potentially fatal consequence of dengue is DHF, where symptoms may appear after 3-7 days of fever, including severe abdominal pain, persistent vomiting, change in body temperature (fever to hypothermia), hemorrhagic manifestations, or change in mental status [16]. An additional severe outcome of dengue comes from the onset of DSS, marked by early signs of shock, restlessness, cold clammy skin, rapid weak pulse, or narrowing pulse pressure [16]. Section 2 reviews some of what is known about DENV-2 strains, American and Asian; discusses strainspecific prevalence and severity and the role of vertical transmission. Section 3 introduces a two-strain model that accounts and explains the role of strain-specific vertical transmission. It also highlights the results of the mathematical analysis and simulations with emphasis on identifying and quantifying necessary, possibly sufficient conditions that guarantee either strain coexistence or competitive exclusion. The role of vertical transmission in providing a competitive advantage of DENV-2 Asian is explored. Section 4, revisits the results of the 
manuscript placing them in the context of dengue dynamics in the Americas. Some of the details of the mathematical analysis are collected in appendices.

\section{Epidemiology of Dengue in Peru Since 2000}

Dengue is a vector borne disease transmitted primarily by the mosquito Ae. aegypti, which has successfully invaded the vast majority of countries in the tropics and sub-tropics [33]. The secondary vector, Ae. albopictus, habituates a considerably larger geographic region with its eggs adapting better to subfreezing temperatures, making it more likely to succeed in areas where it is currently not present like in the U.S. [34, 57]. Vertical transmission of dengue fever (transmission via the vectors' offspring) has been associated with seasonal climate trends and geography (tropics) $[2,7,40]$. Thus, mosquitoes found in highrisk environmental and geographical regions may become more susceptible to infection and transmission of specific dengue serotypes $[7,44,74]$. Previous studies supported the view that vertical transmission of dengue was insignificant [71, 72] albeit recent findings have shown it not to be negligible in captivity and in the wild for Ae. aegypti and Ae. albopictus $[7,18,29,46,65,76]$.

Previous to 2000 only DENV-1 and DENV-2 American genotypes had co-circulated in Peru with neither DHF nor DSS cases reported $[45,56]$. The absence of DHF and DSS in Peru prior to 2000, in the presence of co-circulating DENV-1 and DENV-2 American, has been explained, using the data of experiments carried out in laboratories. These studies identified partial cross-immunity conferred by DENV-1 against DENV-2 American but not conferred against the 2000-2001 invading DENV-2 Asian strain [45]. The successful invasion of DENV-2 Asian has been accompanied by subsequent successful invasions of DENV-3 and DENV-4 [56], and so, Peru is now home to at least four dengue serotypes: DENV-1 and DENV-2 American prevalent in its tropical rain forest prior to 2000, DENV-1, DENV-2 (American and Asian), DENV-3, and DENV-4 serotypes co-circulating in its coastal desert areas including Tumbes, Piura, Lambayeque, La Libertad, and Ancash, following the 20002001 outbreak (illustrated in Figure 2).

The displacement of DENV-2 American by the DENV-2 Asian genotype has also been associated with the appearance of DHF in Cuba, Jamaica, Venezuela, Colombia, Brazil, and Mexico [27, 50, 54, 63]. Table 1 summarizes factors that play a role in the higher levels of pathogenicity associated with DENV-2 Asian infections while collecting biological evidence supporting the greater virulent strength of DENV-2 Asian. The geographical displacement of DENV-2 American by DENV-2 Asian could be modeled, for example, using complex metapopulation models $[11,12]$; and so the question becomes "Can we learn something about dengue displacement dynamics using simpler models?" The rest of the manuscript provides a positive and somewhat satisfactory answer.

\subsection{A Brief Overview of Vertical Transmission}

The standard mode of transmission for dengue is via horizontal transmission (human-tomosquito). The possibility of vertical transmission (transovarial transmission) has gained attention over the last 30 years due, in part, to advances in molecular biology, that allow us to test for the possibility of transovarial transmission. Vertical transmission provides a possible mechanism supporting virus dengue persistence (in nature) in the absence of a recognized host and/or under unfavorable conditions for mosquito activity [42]. While previous studies have been unsuccessful to demonstrate vertical transmission of dengue [71, $72,77]$ other findings have demonstrated that vertical transmission involving Ae. aegypti and Ae. albopictus species is feasible in captivity and in the wild [7, 9, 18, 29, 42, 46, 52, 65, 76].

In field studies, the percentage of natural vertical transmission of DENV from the female to her eggs can be quantified by analyzing the presence of DENV in terms of the minimum infection rates (MIR), which are calculated for vector-bone diseases as the number of (positive pools/total mosquitoes $) \times 1000$. The MIR for dengue virus in Ae. aegypti has been 


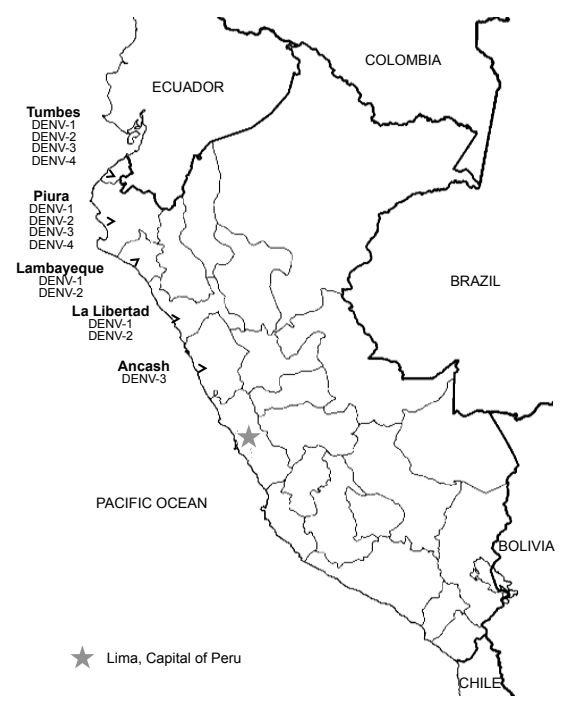

Figure 2: Distribution of Dengue cases in Peru during 2000-2001 (modified from [56]). Prior to 2000, DENV-1 and DENV-2 American were circulating in the country. The 2000-2001, outbreak introduced DENV-2 Asian, DENV-3 and DENV-4 in Peru increasing the potential risk of DHF and DSS in the country.

Table 1: Selected research evidence that supports DENV-2 Asian virulence. Several studies demonstrate the pathogenicity and severity of DENV-2 Asian in disease dynamics. DENV-2 Asian is found to be better equipped for vertical transmission and vector studies have shown that Ae. aegypti are more susceptible to DENV-2 Asian infection. DENV-2 Asian has spread to many countries in South America and the Caribbean Basin. Genetic evidence obtained from mosquito and animal models also highlight the virulence of DENV-2 Asian in these hosts.

Biological Evidence

References

\begin{tabular}{lcc}
\hline \hline The presence of intrinsic genetic mutations in DENV-2 Asian & {$[21,48]$} \\
\hline The lower rate of successful disseminated infection in Ae. aegypti with DENV-2 & {$[5,6]$} \\
American when compared with those associated with DENV-2 Asian & \\
\hline Higher virus output in dendritic cells (five-fold difference) within Ae. aegypti & {$[20]$} \\
mosquitoes for DENV-2 Asian (21\%) versus DENV-2 American (3\%) & \\
\hline Higher vectorial capacity measured by 2 to 65-fold increases in replication of & {$[3]$} \\
DENV-2 Asian in Ae. aegypti midgut when compared to DENV-2 American & \\
\hline DENV-2 Asian is better adapted for vector transmission & {$[5]$} \\
\hline DENV-1 antibodies can protect against DENV-2 American but not DENV-2 & {$[45]$} \\
Asian & {$[6]$} \\
\hline Ae. aegypti is more susceptible to DENV-2 Asian infection & {$[21]$} \\
\hline American genotype reduces dengue virus output in human monocytes and den- & {$[56]$} \\
dritic cells & {$[27]$} \\
\hline DENV-2 Asian takes over Peru, first time with DHF cases & {$[20]$} \\
\hline DENV-2 Asian takes over the Caribbean Basin & \\
\hline Selection of virulent DENV-2 Asian in dendritic cells and mosquitoes & \\
\hline Animal model showed higher viremia and rash in DENV-2 Asian versus DENV-2 & {$[58]$} \\
American &
\end{tabular}

DENV-2 Asian has evolved into several genetic lineages co-circulating with other $[38]$ serotypes 
calculated in the range of $0.18-5[2,6,48,55]$. Detection of DENV specific serotypes using molecular techniques indicates higher infection rates with DENV-2 in Ae. albopicuts (MIR $=26.6$ ) compared to DENV-2 prevalence in Ae. aegypti (MIR $=0.37$ to 1.5$)[2,6,48]$ exist, suggesting that Aedes species display different susceptibilities to dengue virus infections. Additional complications are possible, for example, laboratory results from pooled and individual mosquitoes from regions where dengue is endemic in Peru suggests that Ae. aegypti may be capable of carrying two dengue serotypes simultaneously [14, 49, 51]; a fact that we ignore here, that is, we assume that if possible, its effect is negligible. We assume this because our model focuses on two different strains from the same serotype and replication of DENV-2 Asian in the mosquito vector outcompetes DENV-2 American. Indeed, controlled studies done in a laboratory setting support the hypothesis that higher infection rates exist when Ae. aegypti is exposed to the DENV-2 Asian strain in comparison to DENV-2 American. In a study done by Armstrong and Rico-Hesse [6], Ae. aegypti females derived from mosquitoes captured in Texas and Mexico were exposed via oral feeding to DENV-2 Asian viral isolates from different localities including Venezuela (Mara3) and Peru (Iqt2913). The overall rate of disseminated infection in Ae. aegypti was 27-30\% for all the DENV-2 Asian isolates versus $9-13 \%$ with the American isolates suggesting a higher infection and dissemination efficiency of DENV-2 Asian in Ae. aegypti [5] (shown in Figure 3 (left)). Moreover, viral replication in the midgut turned out to be higher in mosquitoes infected with DENV-2 Asian than in those infected with DENV-2 American (shown in Figure 3 (right)) [5]. Despite the results in these studies, the fact remains that there is still no clear way of identifying the biological mechanisms behind the displacement of DENV-2 American by DENV-2 Asian at the population level (the subject of this manuscript).

Mathematical and statistical models have indeed added to our overall understanding of dengue dynamics even though most have been inspired or driven by the situation in Asia [61]. Dengue is now endemic in more than 100 countries [79] and yet there is no effective vaccine. Furthermore, the discovery of the new DENV-5 serotype (still undetermined) has naturally raised global concerns [62]. The introduction of DENV-2 Asian in northeastern Peru (2011) was responsible for more than 15,000 suspected dengue cases over three weeks, 93 severe cases, and 14 deaths (mostly children under 15). These data provide some evidence that dengue in the Americas may exhibit significant differences due to host, pathogen, climate or geographic heterogeneity [39]. Clearly, worldwide efforts must also monitor the prevalence of DENV genotypes as well as the dynamics of Ae. aegypti mosquito populations (including males $^{1}$ ). Our model considers two different strains from the same serotype and replication of DENV-2 Asian with displacement of DENV-2 American in the population. Here, we are particularly interested in addressing the question of whether or not vertical transmission provides the critical evolutionary advantage needed by DENV-2 Asian to displace DENV-2 American.

\section{Two-strain Dengue Model with Vertical Transmission}

There have been many attempts to gain insights into the dynamics of vector borne diseases (like malaria and dengue). Nishiura (2006) reviews several modeling efforts driven or motivated by dengue. We expand on the simplest framework for dengue presented in [61]. Our model includes progression to DHF but ignores deaths due to DHF (see Figure 4). It also considers two co-circulating genotypes of dengue. Both DHF and DSS, described in the introduction, are severe manifestations of dengue, with associated symptoms being observed following onset of DF (high fever for 3-14 days). DHF and DSS are both thought to be the result of secondary infections with heterologous strains [31, 68]. Our model is based on one specific dengue serotype (DENV-2). While DHF cases are possible in people exposed to one serotype, deaths are low in comparison to DHF cases in a population that

\footnotetext{
${ }^{1}$ To estimate the contribution of vertical transmission to the prevalence of dengue in the mosquito population.
} 

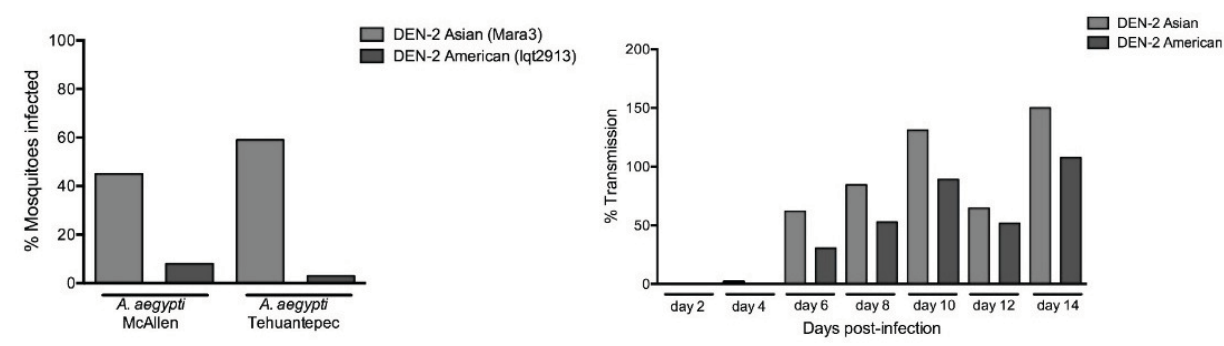

Figure 3: The percentages of mosquitoes infected with DENV-2 Asian (Mara3) versus DENV-2 American (Iqt2913) demonstrate that DENV-2 Asian was more prevalent in the infected populations compared to DENV-2 American (left). Differences in the transmission of DENV-2 Asian and DENV-2 American over 14 days in mosquitoes exposed to the respective viral strains is also shown (right). These figures are adapted from the work of Armstrong and Rico-Hesse [5].

has been previously exposed to a heterotypic serotype (in this case DENV-1, DENV-3, and DENV-4). Thus, sequential heterotypic infections involving more than one serotype lead to an increased disease severity by an antibody-dependent enhancement mechanism (ADE) accounting for the majority of deaths in patients with DHF/DSS [43, 56, 69]. The population at risk of dengue is roughly 3 billion (40 to $60 \%$ of the world population) with 22,000 deaths worldwide $[17,47]$, which is less than the deaths due to seasonal influenza and pneumonia in the U.S. alone (over 40,000) [37]. Even at this rate, mathematical models have ignored deaths due to influenza and yet still obtain relevant model predictions [36]. Here we adopt a similar approach and ignore deaths due to DHF.

We use a SIR model for the host and a SI model for the vector (see [61] for an introduction to this framework in the context of Dengue). The host system includes the class of susceptible humans, $S$, the class of humans infected with American genotype, $D_{A m}$, the class of humans infected with Asian genotype, $D_{A s}$, the class of humans that progress to DHF, $H$, and the class of recovered humans, $R$, (see Figure 4). Vertical transmission is only possible among vectors infected with DENV-2 Asian (see Figure 5). Vectors act as a reservoir for the transmission of dengue from human to vector and do not recover since they are only carriers of the dengue virus. Thus, given their short lifespan [26], the fact that vectors do not recover supports the concept of vertical transmission, that is, if vectors recovered there would be no significant role for vertical transmission.

All of the model parameters are assumed to be (positive) constants. The natural per capita birth/death rate of humans is $\mu$ while the rates at which humans become infected with American or Asian genotype from mosquitoes are $\beta_{A m}$ and $\beta_{A s}$, respectively. The per capita progression rates to DHF is $\alpha$. Further, only individuals infected with DENV-2 Asian can progress to DHF (after presenting DF-like symptoms). The rate at which humans recover from infection from either genotype American, Asian or the extreme DHF stage are $\delta_{A m}, \delta_{A s}$, and $\delta_{H}$, respectively. $N$, the total human population size, is assumed constant since the change in population size is insignificant for a single outbreak, which occurs in less than a year. $M$, the total vector population size, is assumed constant which is biologically reasonable within a single outbreak since the disease has not shown to reduce the lifespan of the vector in the wild with an average lifespan as an adult of roughly 10 days [26]. Moreover, there are more rigorous mathematical approaches based on quassi-steady approximations that incorporate non-constant population sizes [15] however, this is not within the scope of this manuscript.

Individuals diagnosed with DHF are assumed to be hospitalized and so their poten- 


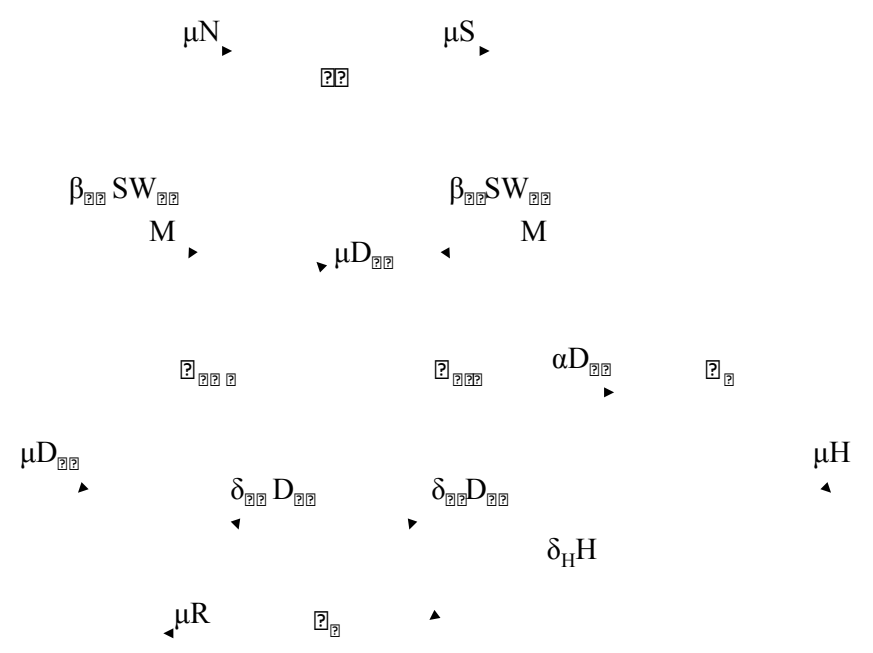

Figure 4: Host model. Susceptible, $S$, infected with DENV-2 American genotype, $D_{A m}$, infected with DENV-2 Asian genotype, $D_{A s}$, progression to DHF, $H$, and recovered, $R$, individuals.

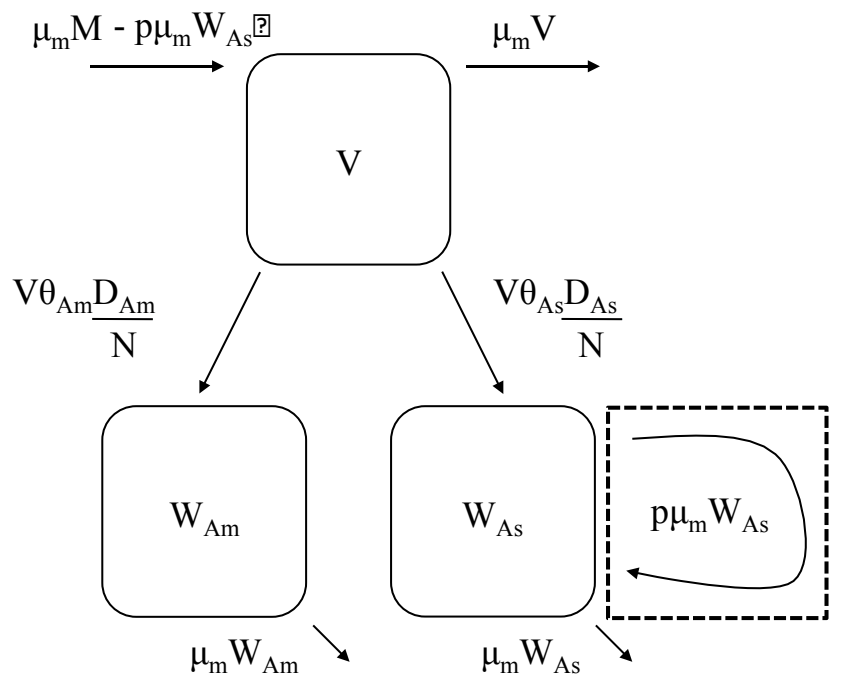

Figure 5: Vector model. Susceptible, $V$, infected with DENV-2 American genotype, $W_{A m}$, and infected with DENV-2 Asian genotype, $W_{A s}$. A certain proportion, $p$, of births confers vertical transmission and enters $W_{a s}$ directly. 
Table 2: Default Parameter Values. Biological parameters may vary across geographic and temporal scales, however, most of the values are taken from related literature or estimated based on common values.

\begin{tabular}{cclc} 
Parameter & Value & Definition (Units) & References \\
\hline$N$ & 1 & Total human population size & {$[26]$} \\
$M$ & 1 & Total vector population size & {$[26]$} \\
$\mu$ & 0.0048 & Human natural birth/death rate (per day) & Estimated \\
$\theta_{A s}$ & 0.8160 & Infectious rate from hosts to vectors, Asian strain (per day) & {$[5]$} \\
$\theta_{A m}$ & 1.6212 & Infectious rate from hosts to vectors, American strain (per day) & {$[5]$} \\
$\beta_{A s}$ & 0.0483 & Infectious rate from vectors to hosts, Asian strain (per day) & {$[21]$} \\
$\beta_{A m}$ & 0.0182 & Infectious rate from vectors to hosts, American strain (per day) & {$[21]$} \\
$\alpha$ & 0.113 & Progression rate to DHF from Asian strain (per day) & {$[78]$} \\
$\mu_{m}$ & 0.0958 & Natural mortality rate of vectors (per day) & {$[64]$} \\
$p$ & 0.103 & Proportion of vectors infected via vertical transmission & {$[46]$} \\
$\delta_{A m}$ & 0.2 & Recovery rate from, American strain (per day) & Estimated \\
$\delta_{A s}$ & 0.2 & Recovery rate from, Asian strain (per day) & Estimated \\
$\delta_{H}$ & 0.2 & Recovery rate from DHF (per day) & Estimated
\end{tabular}

tial to spread dengue is considered to be negligible. In our model, the natural per capita birth/death rate of mosquitoes is $\mu_{m}$, the rate proportion of mosquitoes that are infected through vertical transmission is $p$ (where $p$ in $[0,1]$ ), the rate at which mosquitoes get infected with American genotype is $\theta_{A m}$ and with Asian genotype is $\theta_{A s}$. These parameters and corresponding default values are summarized in Table 2 . The system of nonlinear differential equations used to model the dynamics of two dengue strains in competition is given by

$$
\begin{aligned}
\dot{S} & =\mu N-\frac{\beta_{A m} S W_{A m}}{M}-\frac{\beta_{A s} S W_{A s}}{M}-\mu S, \\
\dot{D}_{A m} & =\frac{\beta_{A m} S W_{A m}}{M}-\left(\delta_{A m}+\mu\right) D_{A m}, \\
\dot{D}_{A s} & =\frac{\beta_{A s} S W_{A s}}{M}-\left(\delta_{A s}+\alpha+\mu\right) D_{A s}, \\
\dot{H} & =\alpha D_{A s}-\left(\delta_{H}+\mu\right) H \\
\dot{R} & =\delta_{A m} D_{A m}+\delta_{A s} D_{A s}+\delta_{H} H-\mu R, \\
\dot{V} & =\mu_{m} M-p \mu_{m} W_{A m}-\frac{V \theta_{A m} D_{A m}}{N}-\frac{V \theta_{A s} D_{A s}}{N}-\mu_{m} V, \\
\dot{W}_{A m} & =\frac{V \theta_{A m} D_{A m}}{N}-\mu_{m} W_{A m}, \\
\dot{W}_{A s} & =\frac{V \theta_{A s} D_{A s}}{N}+p \mu_{m} W_{A s}-\mu_{m} W_{A s} .
\end{aligned}
$$

\subsection{Basic Reproductive Number}

Since $M$ and $N$ are constants, we proceed to rescale the model accordingly, that is, each class is replaced by the proportion of the total respective population. Further, all infected individuals are assumed to recover at the same average per capita rate $\delta$. In the exclusive presence of DENV-2 American, we find that the reproductive number is

$$
\mathcal{R}_{0}^{A m}=\overline{\overline{\beta_{A m}}} \overline{(\delta+\mu)} \cdot \frac{\theta_{A m}}{\mu_{m}},
$$

a dimensionless quantity derived using the next generation operator (see Appendix A). The square root indicates that secondary transmission is a "two-step" process, that is, in order for a human to cause a secondary human infection, a mosquito must be first infected. The average infectious period is $1 /(\delta+\mu)$, and $\beta_{A m}$ is the transmission rate (host to vector). 
Similarly, $\theta_{A m}$ is the transmission rate from vectors to hosts. When $\mathcal{R}_{0}^{A m}>1$, we expect an outbreak of dengue in a population facing only DENV-2 American.

For the case when DENV-2 Asian is the only active genotype, we find that the reproductive number is

$$
\mathcal{R}_{0}^{A s}=\frac{p}{2}+\overline{\frac{p^{2}}{2}+\frac{\beta_{A s}}{(\delta+\alpha+\mu)} \cdot \frac{\theta_{A s}}{\mu_{m}} .}
$$

There are four components to $\mathcal{R}_{0}^{A s}: \theta_{A s} / \mu_{m}$ is the contribution to the reproductive number from infected mosquitoes, the infectious force of mosquitoes times the average time spent in the infectious class; $\beta_{A s} /(\delta+\alpha+\mu)$ is likewise the infectious force of humans times the average time spent in the infectious class; $(p / 2)^{2}$ is the indirect contribution from vertical transmission, that is, from infections caused by mosquitoes that were born with dengue; $p / 2$ is the direct contribution from vertical transmission, infectious mosquitoes create more infectious mosquitoes by giving birth to them. Dengue transmission for DENV-2 American is a "two-step" process due to the absence of vertical transmission.

The basic reproductive number of a model, that considers simultaneously two competing strains is, by definition, the maximum of the two reproductive numbers

$$
\mathcal{R}_{0}=\max \mathcal{R}_{0}^{A m}, \mathcal{R}_{0}^{A s}
$$

In order to assess the role of vertical transmission, we must consider the reproductive number for Asian to be a function of $p$, that is,

$$
\mathcal{R}_{0}^{A s}(p)=\frac{p}{2}+\overline{\frac{p}{2}^{2}+\frac{\beta_{A s}}{(\delta+\alpha+\mu)} \cdot \frac{\theta_{A s}}{\mu_{m}}}
$$

From equation (3), we can compute the critical vertical transmission value $p_{\text {crit }}$ needed for $\mathcal{R}_{0}^{A s}(p)>\mathcal{R}_{0}^{A m}$ given that

$$
\mathcal{R}_{0}^{A s}(0)=\overline{\frac{\beta_{A s}}{(\delta+\alpha+\mu)} \cdot \frac{\theta_{A s}}{\mu_{m}}}<\mathcal{R}_{0}^{A m} .
$$

The critical vertical transmission threshold is therefore defined as

$$
p_{\text {crit }}=\frac{\mathcal{R}_{0}^{A m^{2}}-\mathcal{R}_{0}^{A s}(0)^{2}}{\mathcal{R}_{0}^{A m}} .
$$

Therefore $\mathcal{R}_{0}^{A s}(p)>\mathcal{R}_{0}^{A m}$ whenever $p>p_{\text {crit }}$. Hence, from this definition, we see that in the absence of vertical transmission $(p=0)$ DENV-2 American would dominate only if $\mathcal{R}_{0}^{A s}(0)<$ $\mathcal{R}_{0}^{A m}$. DENV-2 Asian would only dominate (in the absence of vertical transmission) if $\mathcal{R}_{0}^{A s}(0)>1$ and $\mathcal{R}_{0}^{A s}(0)>\mathcal{R}_{0}^{A m}$. In general, for any $p$ greater than $p_{\text {crit }}$, if $p_{\text {crit }}$ is in $[0,1]$ then we have that $\mathcal{R}_{0}^{A s}(p)>\mathcal{R}_{0}^{A m}$, with DENV-2 Asian dominating DENV-2 American thanks to vertical transmission. In general, when $\mathcal{R}_{0}^{A s}\left(p_{\text {crit }}\right)=\mathcal{R}_{0}^{A m}$, if $\mathcal{R}_{0}^{A s}(0) \leq \mathcal{R}_{0}^{A m}$ and $\mathcal{R}_{0}^{A m}>1$, then for $p>p_{\text {crit }}$ we have that

$$
\mathcal{R}_{0}^{A s}(p)>\mathcal{R}_{0}^{A s}(0)>\mathcal{R}_{0}^{A m}
$$

is the vertical transmission condition needed in order for DENV-2 Asian to overtake DENV-2 American.

We describe this in detail in Figure 6 for three cases: (1) $p_{\text {crit }}=0$, (2) $p_{\text {crit }}=0.5$, and (3) $p_{\text {crit }}=1$. In (1), since $\mathcal{R}_{0}^{A s}(0)=\mathcal{R}_{0}^{A m}$ (the solid line), then DENV-2 Asian will dominate under any level of vertical transmission since $\mathcal{R}_{0}^{A s}(p)>\mathcal{R}_{0}^{A m}$ for all $p>0$. Moreover, if $\mathcal{R}_{0}^{A s}(0)>1$ and $\mathcal{R}_{0}^{A s}(0)>\mathcal{R}_{0}^{A m}$, then DENV-2 Asian simply outcompetes DENV-2 American regardless of whether or not vertical transmission is present. Next, in (2), when $\mathcal{R}_{0}^{A s}(0.5)=\mathcal{R}_{0}^{A m}$ (the dotted line), then it is a tie when $\mathcal{R}_{0}^{A s}(0.5)=\mathcal{R}_{0}^{A m}$, but 
any region above the line that is, $\mathcal{R}_{0}^{A s}(p)>\mathcal{R}_{0}^{A m}$, allows DENV-2 Asian to dominate. Lastly, in (3), when $\mathcal{R}_{0}^{A s}(1)=\mathcal{R}_{0}^{A m}$ (the dashed line), $\mathcal{R}_{0}^{A s}(0)$ possibly much less than $\mathcal{R}_{0}^{A m}$, when $\mathcal{R}_{0}^{A s}(p)$ values above the line are enough for DENV-2 Asian to dominate $\left(\mathcal{R}_{0}^{A s}(p)>\mathcal{R}_{0}^{A m}\right)$. In summary, an increase in $p$ leads to a decrease in the threshold required for DENV-2 Asian to outcompete DENV-2 American.

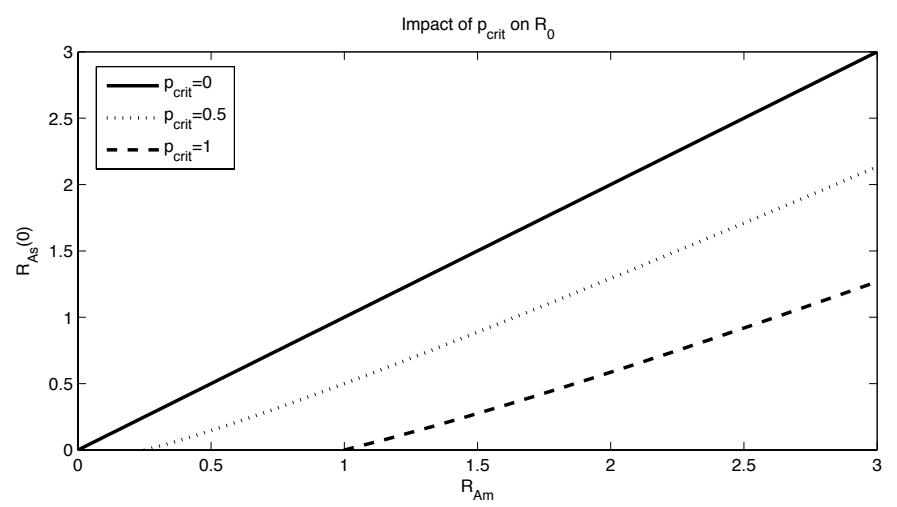

Figure 6: The region where DENV-2 Asian genotype outcompetes DENV-2 American genotype is represented by the area above the line for a fixed $p_{\text {crit }}$. For a fixed $\mathcal{R}_{0}^{A s}(0)$ and $\mathcal{R}_{0}^{A m}$, if $p$ is greater than $p_{\text {crit }}$, graphed, then DENV-2 Asian dominates DENV-2 American. Conversely, if $p=0$ (the solid line), then $\mathcal{R}_{0}^{A s}(p)>\mathcal{R}_{0}^{A m}$ if $\mathcal{R}_{0}^{A s}(0)>\mathcal{R}_{0}^{A m}$. As we increase $p_{\text {crit }}$ to 0.5 (the dotted line), we see that $\mathcal{R}_{0}^{A s}(0)$ can be less than $\mathcal{R}_{0}^{A m}$ and still $\mathcal{R}_{0}^{A s}(p)>\mathcal{R}_{0}^{A m}$. If we further increase $p_{\text {crit }}$ to 1 (the dashed line), then DENV-2 American must be much stronger than DENV-2 Asian in order for DENV-2 American to outcompete DENV-2 Asian.

\subsection{Equilibria}

There are in fact only three equilibria in our system with competitive exclusion precluding strain coexistence. We have a disease free equilibrium (DFE), a DENV-2 American equilibrium $\left(E_{A m}\right)$, and a DENV-2 Asian equilibrium $\left(E_{A s}\right)$. The DFE is

$$
\left(S^{*}=1, D_{A m}^{*}=0, D_{A s}^{*}=0, H^{*}=0, R^{*}=0, V^{*}=1, W_{A m}^{*}=0, W_{A s}^{*}=0\right) .
$$

The DFE always exists and is locally asymptotically stable whenever $\mathcal{R}_{0}<1$, see Appendix A. Further, we make use of a Lyapunov function to show that the DFE is globally asymptotically stable if $\mathcal{R}_{0}<1$, see Appendix C. The equations defining $E_{A m}$ are

$$
\begin{aligned}
S^{*} & =\frac{\mu \beta_{A m}+\mu_{m}(\delta+\mu)}{\theta_{A m}\left(\beta_{A m}+\mu\right)}, & D_{A m}^{*} & =\frac{\mu \mu_{m} \mathcal{R}_{0}^{A m}{ }^{2}-1}{\theta_{A m}\left(\beta_{A m}+\mu\right)}, \\
D_{A s}^{*} & =0, & H^{*} & =0, \\
R^{*} & =1-S^{*}-D_{A m}^{*}, & V^{*} & =1-W_{A m}^{*}, \\
W_{A m}^{*} & =\frac{\mu \mathcal{R}_{0}^{A m}{ }^{2}-1}{\theta_{A m} \mu \mathcal{R}_{0}^{A m}{ }^{2}+\beta_{A m}}, & W_{A s}^{*} & =0 .
\end{aligned}
$$


The $E_{A m}$ only exists if $\mathcal{R}_{0}^{A m}>1$. The equations defining $E_{A s}$ are

$$
\begin{aligned}
S^{*} & =\frac{\theta_{A s} \mu+\mu_{m}(\delta+\alpha+\mu)(1-p)}{\theta_{A s}\left(\beta_{A s}+\mu\right)}, & D_{A m}^{*} & =0, \\
D_{A s}^{*} & =\frac{\mu \mu_{m} \mathcal{R}_{0}^{A s}(0)^{2}+p-1}{\theta_{A s}\left(\beta_{A s}+\mu\right)}, & H^{*} & =\frac{\mu \mu_{m} \alpha \mathcal{R}_{0}^{A s}(0)^{2}+p-1}{\theta_{A s}\left(\beta_{A s}+\mu\right)(\delta+\mu)}, \\
R^{*} & =1-S^{*}-D_{A s}^{*}-H^{*}, & V^{*} & =1-W_{A s}^{*}, \\
W_{A m}^{*} & =0, & W_{A s}^{*} & =\frac{\mu \mu_{m} \mathcal{R}_{0}^{A s}(0)^{2}+p-1}{\mu \mathcal{R}_{0}^{A s}(0)^{2}\left(1+\frac{\mu_{m}}{\beta_{A s}}\right)+1-p} .
\end{aligned}
$$

The $E_{A s}$ only exists if $\mathcal{R}_{0}^{A s}{ }^{2}(0)>1$.

\subsection{Invasion Reproductive Numbers}

The invasion reproductive number (derived in Appendix B) for DENV-2 Asian invading DENV-2 American is

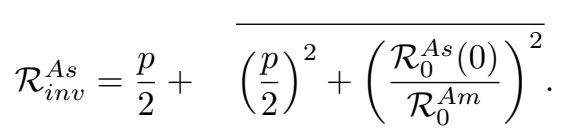

Note, if $p=0$, then the invasion reproductive number is simply the ratio of the DENV-2 Asian in the absence of vertical transmission to DENV-2 American reproductive numbers, as it would be expected from a simple competition model. In order for $E_{A m}$ to be locally asymptotically stable, we must have existence, that is $\mathcal{R}_{0}^{A m}>1$, and strength against invasion, $\mathcal{R}_{i n v}^{A s}<1$. The invasion reproductive number associated with the ability of DENV-2 American to invade DENV-2 Asian is

$$
\mathcal{R}_{i n v}^{A m}=\frac{\mathcal{R}_{0}^{A m}}{\mathcal{R}_{0}^{A s}(0)} \overline{(1-p)} .
$$

We see that if $p=1$ then DENV-2 American could never invade DENV-2 Asian. This is because we would always have a reservoir of DENV-2 Asian, a highly unlikely scenario. We have that $E_{A s}$ is locally asymptotically stable whenever $\mathcal{R}_{0}^{A s}>1$ and $\mathcal{R}_{i n v}^{A m}<1$. Figure 7 a shows how $\mathcal{R}_{i n v}^{A s}<1$ varies as a function of $\mathcal{R}_{0}^{A m}$ and $\mathcal{R}_{0}^{A s}(0)$ for $p=0$. Note that $\mathcal{R}_{\text {inv }}^{A s}=1$ when $\mathcal{R}_{0}^{A m}=\mathcal{R}_{0}^{A s}$. We observe in Figure $7 \mathrm{~b}(p=0.5)$ how the curve has shifted upwards, making it easier for $\mathcal{R}_{\text {inv }}^{A s}>1$. We observe in Figure $7 \mathrm{c},(p=1)$ how the lowest point of the graph is at 1 .

To further illustrate the impact of the invasion reproductive number, we will play out a scenario where DENV-2 American is endemic in a population and DENV-2 Asian is the invasive strain. Suppose DENV-2 American is introduced into a dengue-free region and that $\mathcal{R}_{0}^{A m}>1$, then we would expect an outbreak. In the long-term, DENV-2 American would become and remain endemic in the population, the situation in Peru prior to 2000. The introduction of DENV-2 Asian into the same (now endemic) region may or may not alter the role of DENV-2 American. We know that it is not enough to have $\mathcal{R}_{0}^{A s}>1$ to have an outbreak of DENV-2 Asian because not all of the population is in a susceptible state (some are infected with DENV-2 American). Further, there is possibly a segment of the population that has been exposed to dengue and thus it is protected from DENV-2 Asian. An outbreak of DENV-2 Asian is only possible if we also have that $\mathcal{R}_{i n v}^{A s}>1$, the situation in Peru after 2000. This scenario is shown in Figures 8 and 9. We introduce a few infected people with DENV-2 American in a population of mostly susceptible individuals. The DFE is unstable because $\mathcal{R}_{0}^{A m}>1$ and the outbreak is dominated by DENV-2 American since $\mathcal{R}_{0}^{A m}>\mathcal{R}_{0}^{A s}$ (Figure 8b). DENV-2 American is endemic and DENV-2 Asian dies out in this scenario (Figure 8a). Then we increase $\beta_{A s}$ so that $\mathcal{R}_{i n v}^{A s}>1$. Another outbreak is triggered, but this time it is dominated by DENV-2 Asian (Figure 9b). The population 


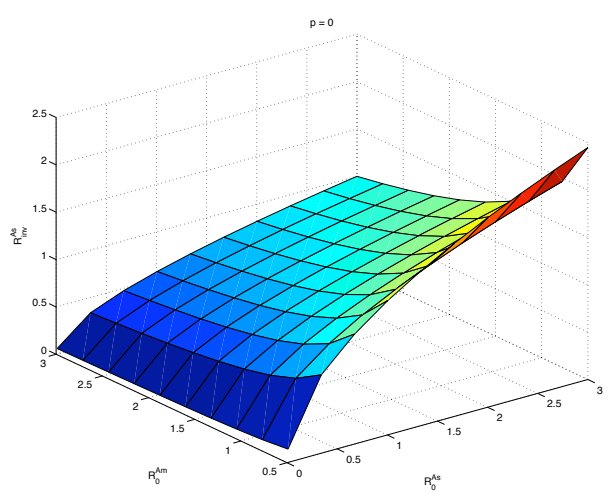

(a) $p=0$, DENV-2 Asian invades if $\mathcal{R}_{0}^{A s}(0)>\mathcal{R}_{0}^{A m}$.

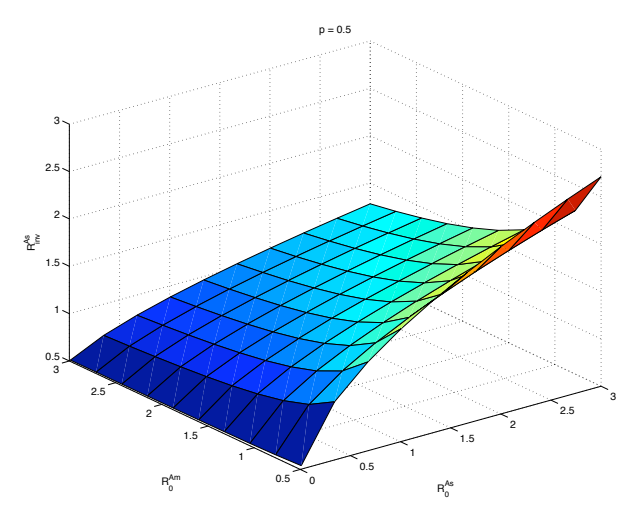

(b) Increasing $p$ to 0.5 , facilitates DENV-2 Asian to invade as noted by the upward shift of the graph.

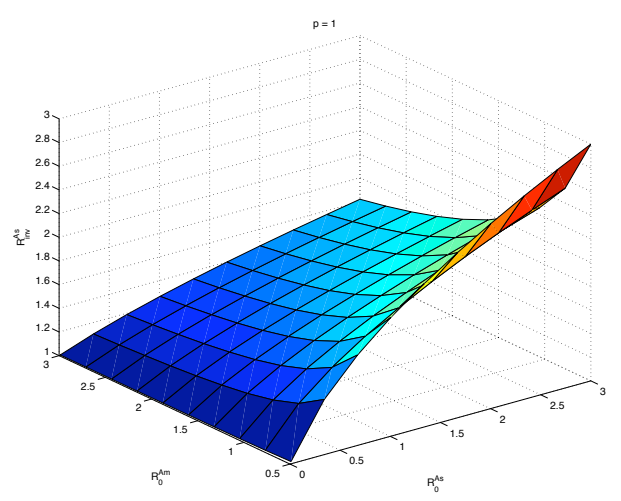

(c) As $p$ is further increased to 1 , it becomes much easier for DENV-2 Asian to invade.

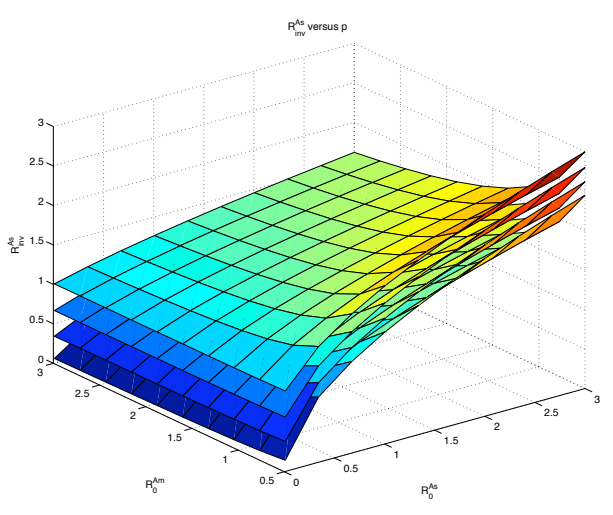

(d) Increasing $p$ shifts upward the manifold for $\mathcal{R}_{i n v}^{A s}$ as a function of $\mathcal{R}_{0}^{A s}(0)$ and $\mathcal{R}_{0}^{A m}$.

Figure 7: Invasion reproductive number for DENV-2 Asian into DENV-2 American. As $p$ is increased, it becomes easier for DENV-2 Asian to invade DENV-2 American, represented by the upward shift of the graph. 
started near an endemic level of DENV-2 American $\left(E_{A m}\right)$, but now the DENV-2 Asian tends to an endemic level $\left(E_{A s}\right)$ while DENV-2 American tends to zero (Figure 9a).
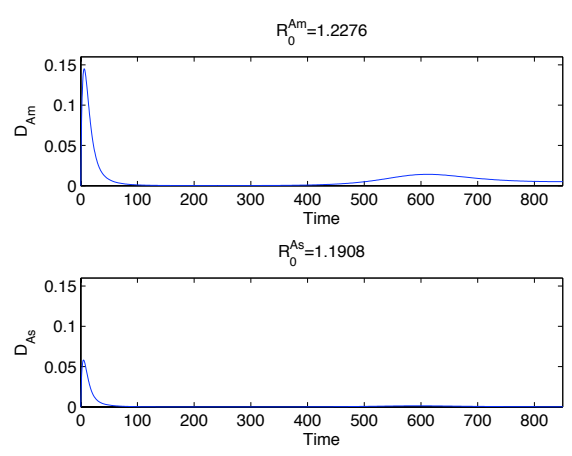

(a) First outbreak is dominated by DENV-2 American since $\mathcal{R}_{0}^{A m}>\mathcal{R}_{0}^{A s}$.

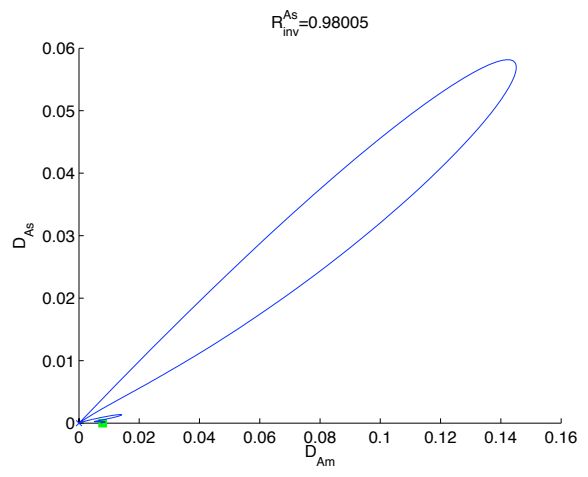

(b) After the initial outbreak, there is an endemic level of DENV-2 American.

Figure 8: If $\mathcal{R}_{0}^{A s}<1, \mathcal{R}_{0}^{A m}>1$, then $E_{A m}$ is stable as DENV-2 American tends to some endemic level and DENV-2 Asian tends to zero (a). The phase portrait shows the relative prevalence of each DENV-2 strain (b). The outbreak starts near the origin and eventually tends towards $E_{A m}$ (square).

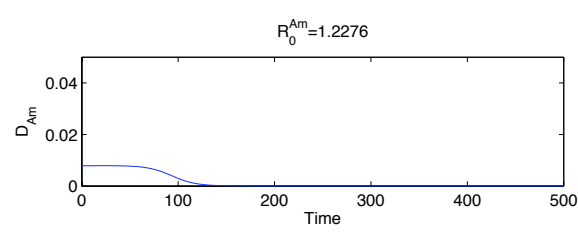

$\mathrm{R}_{0}^{\mathrm{As}}=1.8341$

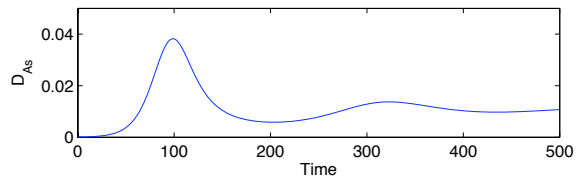

(a) Second outbreak is dominated by DENV-2 Asian since $\mathcal{R}_{0}^{A s}>\mathcal{R}_{0}^{A m}$.

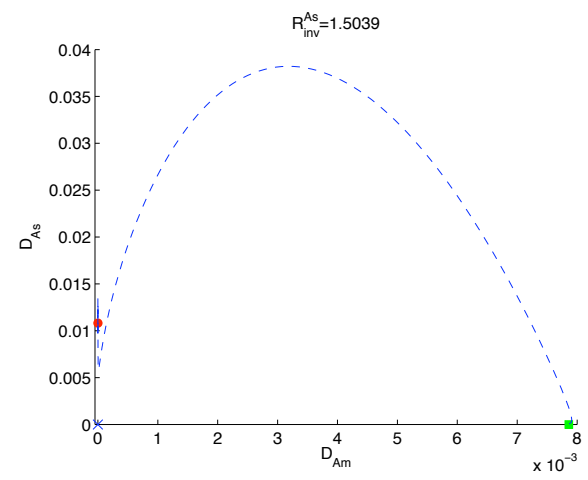

(b) After the initial outbreak, there is an endemic level of DENV-2 Asian.

Figure 9: If the outbreaks starts near $E_{A m}$ and $\mathcal{R}_{i n v}^{A s}>1$, then DENV-2 American tends to zero and DENV-2 Asian tends to an endemic level (a). The phase portrait shows the relative prevalence of each DENV-2 strain (b). The outbreak starts near $E_{A m}$ (square) and eventually tends towards $E_{A s}$ (circle).

Similarly, we can illustrate how $\mathcal{R}_{\text {inv }}^{A m}$ varies as a function of $\mathcal{R}_{0}^{A m}$ and $\mathcal{R}_{0}^{A s}(0)$ for fixed $p$, see Figure 10. The top most manifold corresponds to $p=0$. As $\mathcal{R}_{0}^{A m}$ increases, $\mathcal{R}_{i n v}^{A m}$ increases. When we increase $p$, the manifold lowers and flattens out until the point when it is flat at $p=0$. At this point, DENV-2 American is no longer able to invade DENV-2 Asian as no value for $\mathcal{R}_{0}^{A m}$ will make $\mathcal{R}_{\text {inv }}^{A m}>1$

\section{Discussion}

While Bernoulli was perhaps the first to mathematically model a disease in 1760 [11, 59], it took more than a century before Ross introduced the first models to study the mosquitoes spreading Malaria in the 1900s [11, 28, 70]. Over a century after the groundbreaking work of Ross, models are still being used to study Malaria [53], Dengue [67, 19, 28, 61], and 


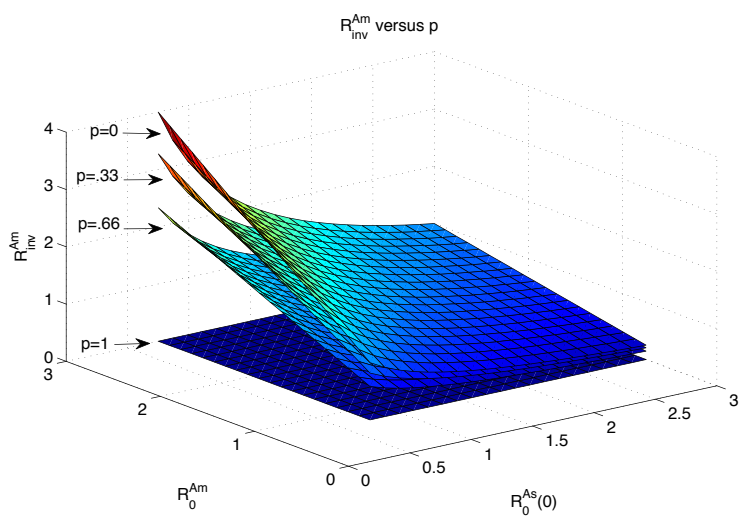

Figure 10: As $p$ increases, it becomes more difficult for DENV-2 American to invade DENV-2 Asian, as indicated by the manifold decreasing and flattening out. Ultimately, when $p=1$, DENV-2 American is no longer able to invade as represented by the bottom most, flat graph.

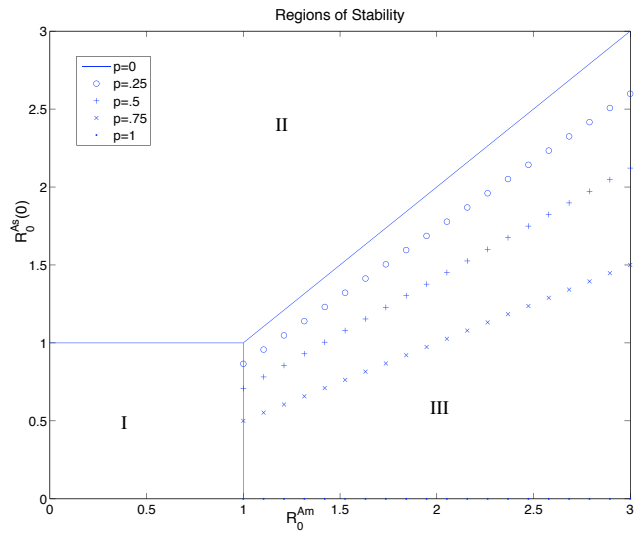

Figure 11: There are three regions of stability. The DFE is stable when $\mathcal{R}_{0}^{A s}<1$ and $\mathcal{R}_{0}^{A m}<1$, Region I. $E_{A s}$ is stable in Region II (when $p=0$ ) if $\left(\mathcal{R}_{0}^{A s}{ }^{2}(0)>1\right.$ and $\mathcal{R}_{0}^{A s}(0)>\mathcal{R}_{0}^{A m}$, in this case $E_{A m}$ will be unstable. In Region III, where $p>0, E_{A m}$ is stable only if $\mathcal{R}_{0}^{A m}>1$ and $\mathcal{R}_{0}^{A s}(p)<\mathcal{R}_{0}^{A m}$, in this case $E_{A s}$ becomes unstable. However, $E_{A s}$ is stable if $\left(\mathcal{R}_{0}^{A s}{ }^{2}(p)>1\right.$ and $\mathcal{R}_{0}^{A s}(p)>\mathcal{R}_{0}^{A m}$, then $E_{A m}$ becomes unstable. You can see that Region II can expand thanks to vertical transmission. 
other vector-borne disease such as West Nile Virus [10, 22]. However, the role of vertical transmission in vector-borne models has rarely been studied, but see the contribution by Busenberg and Cooke [13], whereas HIV has become the canonical example of vertical transmission for a sexually-transmitted disease [4, 8, 73]. Similar mathematical models to the one presented here have been developed, [1, 25, 61], suggesting the importance of vertical transmission to the persistence of dengue where there is a widely fluctuating environment and diapause. Our research shows that the landscape is changing by the introduction of a competing strain through vertical transmission into an endemic area, which in turn, makes dengue more likely to both spread in an area without prior infection or invade an area dominated by a "native" strain.

Our model was inspired by the 2000-2001 dengue epidemic in the northwest region of Peru, the first time that DHF cases were observed in this region following the introduction of DENV-2 Asian [56]. Despite the endemic presence of both DENV-1 and DENV-2 American in Peru, no DHF cases were previously detected, possibly explained by the cross-immunity "conferred" by DENV-1 against DENV-2 American strain and not the Asian [45]. Moreover, data from the 2000-2001 outbreak in Peru showed that DENV-2 Asian displaced DENV-2 American as observed by Restriction Fragment Length Polymorphism (RFLP) analysis and corroborated by partial sequence analysis [56]. Displacement of DENV-2 American by the DENV-2 Asian has previously been documented in the Americas [30, 63]. While co-infection with two serotypes is feasible in both the mosquito and human population [14, 49], the fact is that the proportion of individuals reported carrying two or more serotypes has always been insignificant [51]. Moreover, when DENV-2 Asian and DENV-2 American co-circulate DENV-2 Asian would outcompete DENV-2 American [5, 6]. Co-infection with a different serotype should not be ignored as it is biologically possible and it will be explored in a future mathematical model.

Invasion reproductive numbers generally arise in the context of two species competing for the same niche leading to competitive exclusion [80]. In an area where neither DENV-2 Asian nor American is present, the basic reproductive number is simply the maximum of the two, strain specific, reproductive numbers

$$
\begin{aligned}
& \mathcal{R}_{0}=\max \mathcal{R}_{0}^{A m}, \mathcal{R}_{0}^{A s} \\
& \mathcal{R}_{0}^{A m}=\overline{\frac{\beta_{A m}}{(\delta+\mu)} \cdot \frac{\theta_{A m}}{\mu_{m}},} \\
& \mathcal{R}_{0}^{A s}(p)=\frac{p}{2}+\overline{\frac{p^{2}}{2}+\frac{\beta_{A s}}{(\delta+\alpha+\mu)} \cdot \frac{\theta_{A s}}{\mu_{m}},}
\end{aligned}
$$

where we note $\mathcal{R}_{0}^{A s}(p)$ is a strictly increasing function of the vertical transmission parameter, $p$. In a completely susceptible population, vertical transmission can improve the chances of a DENV-2 Asian outbreak $\left(\mathcal{R}_{0}^{A s}(0)<1\right.$ and $\mathcal{R}_{0}^{A s}\left(p_{\text {crit }}\right)>1$ with $p_{\text {crit }}$ in $\left.[0,1]\right)$. Furthermore, in an area already endemic with DENV-2 American, vertical transmission facilitates the invasion, and replacement by DENV-2 Asian provided the invasion reproductive number is greater than one

$$
\mathcal{R}_{i n v}^{A s}=\frac{p}{2}+\overline{\frac{p}{2}^{2}+{\frac{\mathcal{R}_{0}^{A s}(0)}{\mathcal{R}_{0}^{A m}}}^{2}}
$$

again a situation when vertical transmission can help. Similarly, if DENV-2 Asian is endemic in a region then vertical transmission can make it more difficult for DENV-2 American to invade since

$$
\mathcal{R}_{i n v}^{A m}=\frac{\mathcal{R}_{0}^{A m}}{\mathcal{R}_{0}^{A s}(0)} \quad \overline{(1-p)} .
$$

Our results show that even a low probability of vertical transmission can have a major impact on the long term dynamics of dengue fever. For two competing strains, vertical 
transmission can make a difference between failed outbreaks or invasions, as well as the ability to become endemic in a population. Our model results highlight the importance of vertical transmission in a dengue outbreak as well as the importance of epidemiological surveillance that accounts for molecular genotyping. Routine detection of the virus in both mosquitoes and hosts in endemic areas with dengue will be valuable in order to prevent major outbreaks and gauge the severity of the response that is required to combat any potential outbreak.

\section{Acknowledgements}

The authors are solely responsible for the views and opinions expressed in this research; it does not necessarily reflect the ideas and/or opinions of the funding agencies or Arizona State University. This publication was made possible by grant number 1R01GM100471-01 from the National Institute of General Medical Sciences (NIGMS) at the National Institutes of Health. Its contents do not necessarily represent the official views of NIGMS. The authors would also like to acknowledge Kamal Barley for assistance with graphical enhancements.

\section{A $\quad \mathcal{R}_{0}$ via Next Generation Operator}

We use the next generation operator to calculate the basic reproductive number. This method has several advantages over other methods especially in the context of vector-borne diseases, see [75] or [24] for a more complete discussion and proof of the method. In this section we outline how this method is applied. First we must identify the infected classes: $D_{A m}, D_{A s}, W_{A m}, W_{A s}$, and $H$. Then we must identify the "new" infections. Infections coming into $D_{A m}, D_{A s}, W_{A m}$, and $W_{A s}$ are new while infections coming into $H$ are "old" since individuals must first be infected via $D_{A s}$. Then we form two vectors, $\mathcal{F}$ which consist of only the new infection terms, and $\mathcal{V}$ that is the negation of the remaining terms in our infected classes, that is

$$
\mathcal{F}-\mathcal{V}=\begin{gathered}
\dot{D_{A m}} \\
\dot{D_{A s}} \\
W_{A m}^{\dot{m}} \\
\dot{W_{A s}} \\
\dot{H}
\end{gathered}
$$

Then

$$
\begin{aligned}
& \frac{\beta_{A m} S W_{A m}}{M} \quad(\delta+\mu) D_{A m} \\
& \frac{\beta_{A s} S W_{A s}}{M} \\
& \mathcal{F}=\begin{array}{c}
\frac{\theta_{A m} V D_{A m}}{N} \\
\frac{\theta_{A s} V D_{A s}}{N}+p \mu_{m} W_{A s}
\end{array} \\
& \text { and } \quad \mathcal{V}=\quad \mu_{m} W_{A m} \text {. } \\
& 0 \\
& (\delta+\mu) H-\alpha D_{A s}
\end{aligned}
$$

Next we calculate the Jacobian Matrices

$$
\mathbf{F}=\begin{array}{ccccccccccc}
0 & 0 & \frac{\beta_{A m} S}{M} & 0 & 0 & & \delta+\mu & 0 & 0 & 0 & 0 \\
0 & 0 & 0 & \frac{\beta_{A s} S}{M} & 0 \\
\frac{\theta_{A m} V}{N} & 0 & 0 & 0 & 0 \\
0 & \frac{\theta_{A m} V}{N} & 0 & p \mu_{m} & 0 & & 0 & & \\
0 & 0 & 0 & 0 & 0 & & 0 & 0 & \mu_{m} & 0 & 0 \\
0 & 0 & 0 & -\alpha & 0 & 0 & \delta+\mu
\end{array} .
$$


Next we must evaluate the Jacobian matrices at the disease free equilibrium (Equation 5), keeping in mind that we have normalized both the human and mosquito populations. Then it only remains to find the eigenvalues of $\mathbf{F} \mathbf{V}^{-1}$ since the basic reproductive number is the spectral radius, or largest eigenvalue of the next generation matrix

$$
\mathcal{R}_{0}=\rho\left(\mathbf{F V}^{-1}\right)
$$

Carrying out the calculation yields two candidates (the other eigenvalues are zero or strictly smaller than these candidates)

$$
\mathcal{R}_{0}^{A m}=\overline{\frac{\beta_{A m}}{(\delta+\mu)} \cdot \frac{\theta_{A m}}{\mu_{m}}} \quad \text { and } \quad \mathcal{R}_{0}^{A s}=\frac{p}{2}+\overline{\frac{p}{2}^{2}+\frac{\beta_{A s}}{(\delta+\alpha+\mu)} \cdot \frac{\theta_{A s}}{\mu_{m}}}
$$

where

$$
\mathcal{R}_{0}=\max \mathcal{R}_{0}^{A m}, \mathcal{R}_{0}^{A s}
$$

as required.

\section{B Invasion Reproductive Numbers}

To find the invasion reproductive number, we follow the same methodology as in finding the basic reproductive number. However, instead of assuming the entire population is susceptible to infection, we assume that one strain is already established, endemic, in the population. Then "new" infections are only those infections associated with the invading strain. Then for Asian invading American, only classes $D_{A s}, W_{A s}$, and $H$ are of interest with new infections in the $D_{A s}$ and $W_{A s}$ classes:

$$
\mathcal{F}=\begin{gathered}
\frac{\beta_{A s} S W_{A s}}{M} \\
\frac{\theta_{A s} V D_{A s}}{N}+p \mu_{m} W_{A s} \\
0
\end{gathered} \quad \text { and } \quad \mathcal{V}=\begin{gathered}
(\delta+\alpha+\mu) D_{A s} \\
\mu_{m} W_{A s} \\
(\delta+\mu) H-\alpha D_{A s}
\end{gathered}
$$

Next we calculate the Jacobian Matrices

$$
\mathbf{F}=\begin{array}{ccl}
0 & \frac{\beta_{A s} S}{M} & 0 \\
\frac{\theta_{A s} V}{N} & p \mu_{m} & 0 \\
0 & 0 & 0
\end{array} \quad \text { and } \quad \mathbf{V}=\begin{array}{cccc}
\delta+\alpha+\mu & 0 & 0 \\
0 & \mu_{m} & 0 \\
-\alpha & 0 & \delta+\mu
\end{array} .
$$

Next we evaluate these Jacobian matrices at the American equilibrium (Equation 6), since we assumed strain 1 was endemic prior to the introduction of Asian. Then it only remains to find the eigenvalues of $\mathbf{F V}^{-1}$ yields only one candidate

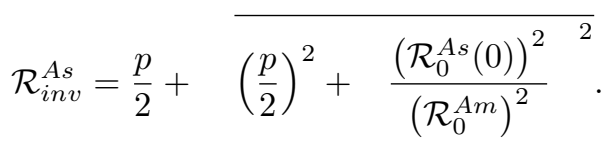

Similarly for American invading Asian, $D_{A m}$ and $W_{A m}$ are the classes of interest.

$$
\mathcal{F}=\frac{\begin{array}{c}
\beta_{A m} S W_{A m} \\
M
\end{array}}{\frac{\theta_{A m} V D_{A m}}{N}} \quad \text { and } \quad \mathcal{V}=\begin{gathered}
(\delta+\mu) D_{A m} \\
\mu_{m} W_{A m}
\end{gathered} .
$$

Next we calculate the Jacobian Matrices

$$
\mathbf{F}=\begin{array}{cc}
0 & \frac{\beta_{A m} S}{M} \\
\frac{\theta_{A m} V}{N} & 0
\end{array} \quad \text { and } \quad \mathbf{V}=\begin{array}{cc}
\delta+\mu & 0 \\
0 & \mu_{m}
\end{array}
$$

and we evaluate these Jacobian matrices at the Asian equilibrium (Equation 7), since we assumed strain 2 was endemic. Then the dominant eigenvalue of $\mathbf{F V}^{-1}$ is

$$
\mathcal{R}_{i n v}^{A m}=\frac{\mathcal{R}_{0}^{A m^{2}}(1-p)}{\mathcal{R}_{0}^{A s}(0)^{2}} .
$$




\section{Global stability of $\mathcal{R}_{0}$ via the Lyapunov-LaSalle Theorem}

Using the rescaled system of equations, let

$$
L=D_{A m}+D_{A s}+\frac{\beta_{A m}}{\mu_{m}} W_{A m}+\frac{\beta_{A s}}{\mu_{m}} W_{A s} .
$$

Clearly $L$ is positive definite and radially unbounded. The orbital derivative of $L$ is

$$
\begin{aligned}
\dot{L}= & \beta_{A m} W_{A m} S-\left(\delta_{A m}+\mu\right) D_{A m}+\beta_{A s} W_{A s} S-\left(\delta_{A m}+\mu\right) D_{A m} \\
& +\theta_{A m} D_{A m} V-\mu_{m} W_{A m}+\theta_{A s} D_{A s} V-\mu_{m} W_{A s}+p \mu_{m} W_{A s} \\
=- & \beta_{A m} W_{A m}(1-S)-\left(\delta_{A m}+\mu\right) D_{A m}\left(1-\mathcal{R}_{0}^{A m} V\right) \\
& -\beta_{A s} W_{A s}\left(1-S-p W_{A s}\right)-\left(\delta_{A s}+\mu\right) D_{A s}\left(1-\mathcal{R}_{0}^{A s}(0) V\right) .
\end{aligned}
$$

If $\mathcal{R}_{0}<1$, then clearly $\dot{L} \leq 0$. Next we must show that the DFE is the maximal invariant subspace of $\dot{L}=0$. From above we have four conditions that must be simultaneously satisfied for $\dot{L}=0$ :

$$
\begin{aligned}
W_{A m}(1-S) & =0, \\
W_{A s}\left(1-S-p W_{A s}\right) & =0, \\
D_{A m}\left(1-\mathcal{R}_{0}^{A m} V\right) & =0, \\
D_{A s}\left(1-\mathcal{R}_{0}^{A s}(0) V\right) & =0 .
\end{aligned}
$$

Equation 10 implies that either $W_{A m}=0$ or $S=1$. Suppose $W_{A m} \neq 0$, then $S=1$ and $\dot{S}=0$ but

$$
\dot{S}=-\beta_{A m} W_{A m}-\beta_{A s} W_{A s}<0,
$$

which is a contradiction, thus $W_{A m}=0$. From Equation 12 either $D_{A m}=0$ or $V=1 / \mathcal{R}_{0}^{A m}$. Suppose $D_{A m} \neq 0$, since $\mathcal{R}_{0}^{A m}<1, V>1$ which is impossible since $V \in[0,1]$. Therefore $D_{A m}=0$. Similarly, Equation 13 implies that either $D_{A s}=0$ or $V=1 / \mathcal{R}_{0}^{A s}(0)$, but if $\mathcal{R}_{0}^{A s}<1$, then $\mathcal{R}_{0}^{A s}(0)<1$ and we conclude $D_{A s}=0$. Finally, Equation 11 implies that either $W_{A s}=0$ or $S=1-p W_{A s}>0$ if $p<1$. Suppose $W_{A s} \neq 0$. Since $D_{A s}=0, \dot{D}_{A s}=0$, and

$$
\dot{D}_{A s}=\beta_{A s} S W_{A s}>0,
$$

thus we conclude $W_{A s}=0$. Also note that since $\dot{D}_{A s}=0, \dot{H}=-\left(\delta_{H}+\mu\right) H$ which implies $H \rightarrow 0$, thus the maximal invariant set is the DFE. Hence, by the Lyapunov-LaSalle Theorem, the DFE is locally asymptotically stable.

\section{References}

[1] B. Adams and M. Boots (2010). How important is vertical transmission in mosquitoes for the persistence of dengue? Insights from a mathematical model. Epidemics, 2(1):110.

[2] M. R. Akbar, R. Agoes, T. Djatie, and S. Kodyat (2009). PCR Detection of Dengue Transovarial Transmissibility in Aedes aegypti in Bandung, Indonesia. Proc ASEAN Congr Trop Med Parasitol, 3:84-89.

[3] J. R. Anderson and R. Rico-Hesse (2006). Aedes aegypti vectorial capacity is determined by the infecting genotype of dengue virus. Am J Trop Med Hyg, 75(5):886-892.

[4] R. M. Anderson (1988). The role of mathematical models in the study of HIV transmission and the epidemiology of AIDS. JAIDS-J Acq Imm Def, 1(3):241-256. 
[5] P. M. Armstrong and R. Rico-Hesse (2001). Differential susceptibility of Aedes aegypti to infection by the American and Southeast Asian genotypes of dengue type 2 virus. Vector-Borne Zoonot, 1(2):159-168.

[6] P. M. Armstrong and R. Rico-Hesse (2003). Efficiency of dengue serotype 2 virus strains to infect and disseminate in Aedes aegypti. Am J Trop Med Hyg, 68(5):539-544.

[7] N. Arunachalam, S. C. Tewari, V. Thenmozhi, R. Rajendran, R. Paramasivan, R. Manavalan, K. Ayanar, and B.K. Tyagi (2008). Natural vertical transmission of dengue viruses by Aedes aegypti in Chennai, Tamil Nadu, India. Indian J Med Res, 127:395.

[8] S. M. Blower and H. Dowlatabadi (1994). Sensitivity and uncertainty analysis of complex models of disease transmission: an HIV model, as an example. International Statistical Review/Revue Internationale de Statistique, 229-243.

[9] C.F. Bosio, R.E. Thomas, P. R. Grimstad, and K.S. Rai (1992). Variation in the efficiency of vertical transmission of dengue-1 virus by strains of Aedes albopictus (Diptera: Culicidae). J Med Entomol, 29(6):985-989.

[10] C. Bowman, A. B. Gumel, P. Van den Driessche, J. Wu, and H. Zhu (2005). A mathematical model for assessing control strategies against West Nile virus. B Math Biol, 67(5):1107-1133.

[11] F. Brauer and C. Castillo-Chavez (2012). Mathematical models in population biology and epidemiology. Springer.

[12] F. Brauer and C. Castillo-Chavez (2013). Mathematical Models for Communicable Diseases, volume 84 of CBMS-NSF regional conference series in applied mathematics. Society for Industrial and Applied Mathematics, Philadelphia, PA.

[13] S. Busenberg and K. Cooke (1993). Vertically Transmitted Diseases. Springer.

[14] O. Cáceres (2003). Detección rápida de los serotipos del virus dengue en el mosquito Aedes aegypti. Revista Peruana de Medicina Experimental y Salud Publica, 20(3):156158.

[15] C. Castillo-Chavez and B. Song (2004). Dynamical models of tuberculosis and their applications. Math Biosci Eng, 1(2), 361-404.

[16] CDC (2009). Dengue and Dengue Hemorrhagic Fever Information for Health Care Practitioners.

[17] CDC (2010). Report Suggests Nearly 5 Percent Exposed to Dengue Virus in Key West.

[18] A. B. Cecílio, E.S. Campanelli, K.P. R. Souza, L. B. Figueiredo, and M. E. Resende (2009). Natural vertical transmission by Stegomyia albopicta as dengue vector in Brazil. Braz J Biol, 69(1):123-127.

[19] G. Chowell and F. Sanchez (2006). An Outbreak of Dengue in Mexico, 2003: Quantifying the role of interventions. $J$ Environ Health, 68(10):40-44.

[20] R. Cologna, P. M. Armstrong, and R. Rico-Hesse (2005). Selection for virulent dengue viruses occurs in humans and mosquitoes. J Virol, 79(2):853-859.

[21] R. Cologna and R. Rico-Hesse (2003). American genotype structures decrease dengue virus output from human monocytes and dendritic cells. J Virol, 77(7):3929-3938.

[22] G. Cruz-Pacheco, L. Esteva, J. A. Montano, and C. Vargas (2004). A Mathematical Model for the Dynamics of West Nile Virus. 
[23] V. Deubel, R. M. Kinney, and D. W. Trent (1986). Nucleotide sequence and deduced amino acid sequence of the structural proteins of dengue type 2 virus, Jamaica genotype. Virology, 155(2):365-377.

[24] O. Diekmann, J. A. P. Heesterbeek, and J. A. Metz (1990). On the definition and the computation of the basic reproduction ratio $\mathrm{R} 0$ in models for infectious diseases in heterogeneous populations. J Math Biol, 28(4):365-382.

[25] L. Esteva and C. Vargas (2000). Influence of vertical and mechanical transmission on the dynamics of dengue disease. Math Biosci, 167(1):51-64.

[26] D. A. Focks, N. Alexander, E. Villegas, and World Heath Organization. (2006). Multicountry study of aedes aegypti pupal productivity survey methodology: findings and recommendations.

[27] J. E. Foster, S. N. Bennett, C. V. Carrington, H. Vaughan, and W. O. McMillan (2004). Phylogeography and molecular evolution of dengue 2 in the caribbean basin, 1981-2000. Virology, 324(1):48-59.

[28] A. B. Gumel, C. Castillo-Chavez, R. E. Mickens, and D. P. Clemence (2006). Mathematical Studies on Human Disease Dynamics: Emerging Paradigms and Challenges. AMS-IMS-SIAM Joint Summer Research Conference on Modeling the Dynamics of $\mathrm{Hu}$ man Diseases: Emerging Paradigms and Challenges, July 17-21, 2005, Snowbird, Utah (Vol. 410). American Mathematical Soc.

[29] J. Gunther, J. P. Martínez-Muñoz, D. G. Pérez-Ishiwara, and J. Salas-Benito (2007). Evidence of vertical transmission of dengue virus in two endemic localities in the state of Oaxaca, Mexico. Intervirology, 50(5):347-352.

[30] M. G. Guzman, V. Deubel, J.L. Pelegrino, D. Rosario, M. Marrero, C. Sariol, and G. Kouri (1995). Partial nucleotide and amino acid sequences of the envelope and the envelope/nonstructural protein-1 gene junction of four dengue-2 virus strains isolated during the 1981 Cuban epidemic. Am J Trop Med, 52(3):241-246.

[31] S. B. Halstead (1988). Pathogenesis of dengue: challenges to molecular biology. Science, 239(4839):476-481.

[32] S. B. Halstead, N. T. Lan, T. N. Myint, T. Shwe, A. Nisalak, S. Kalyanarooj, S. Nimmannitya, S. Soegijanto, D. W. Vaughn, and T. P. Endy (2002). Dengue hemorrhagic fever in infants: research opportunities ignored. Emerg Infec Dis, 8(12):1474-1479.

[33] E. Harris, E. Videa, L. Pérez, E. Sandoval, Y. Téllez, M. L. Pérez, R. Cuadra, J. Rocha, W. Idiaquez, R. Alonso, M. A. Delgado, L. A. Campo, F. Acevedo, A. Gonzalez, J. J. Amador, and A. Balmaseda (2000). Clinical, epidemiologic, and virologic features of dengue in the 1998 epidemic in Nicaragua. Am J Trop Med, 63(1):5-11.

[34] W. A. Hawley, P. Reiter, R. S. Copeland, C. B. Pumpuni, and G. B. Craig (1987). Aedes albopictus in North America: probable introduction in used tires from northern Asia. Science, 236(4805):1114-1116.

[35] N. Hernandez-Ceron, Z. Feng, and C. Castillo-Chavez (2013). Discrete epidemic models with arbitrary stage distributions and applications to disease control. B Math Biol, 75(10):1716-1746.

[36] M. A. Herrera-Valdez, M. Cruz-Aponte, and C. Castillo-Chavez (2011). Multiple outbreaks for the same pandemic: Local transportation and social distancing explain the different "waves" of A-H1N1pdm cases observed in Mexico during 2009. Math Biosci Eng, 8(1):21-48. 
[37] D. L. Hoyert and J. Xu (2012). Deaths: preliminary data for 2011. National vital statistics reports, 61(6):1-51.

[38] E. Huhtamo, G. Comach, G. Sierra, D. E. Camacho, T. Sironen, O. Vapalhti, and N. Y. Uzcátegui (2013). Diversity and composition of dengue virus type 2 in venezuela. Epidemiol Infect, 141(09):1816-1822.

[39] IFRRS (2011). Peru and Bolivia: Dengue outbreak. International Federation of Red Cross and Red Crescent Societies.

[40] V. Joshi, D. T. Mourya, and R. C. Sharma (2002). Persistence of dengue-3 virus through transovarial transmission passage in successive generations of Aedes aegypti mosquitoes. Am J Trop Med, 67(2):158-161.

[41] M. Kermark and A. G. Mckendrick (1927). Contributions to the mathematical theory of epidemics. part i. In Proc. R. Soc. A (Volume 115, No. 5, pp. 700-721).

[42] M. M. Khin and K. A. Than (1983). Transovarial transmission of dengue 2 virus by Aedes aegypti in nature. Am J Trop Med, 32(3):590-594.

[43] S. C. Kliks, A. Nisalak, W.E. Brandt, L. Wahl, and D.S. Burke (1989). Antibodydependent enhancement of dengue virus growth in human monocytes as a risk factor for dengue hemorrhagic fever. Walter Reed Army Inst. of Research, Washington, DC, Dept of Virus Research.

[44] T. B. Knox, B. H. Kay, R. A. Hall, and P. A. Ryan (2003). Enhanced vector competence of Aedes aegypti (Diptera: Culicidae) from the Torres Strait compared with mainland Australia for dengue 2 and 4 viruses. J Med Entomol, 40(6):950-956.

[45] T. J. Kochel, D. M. Watts, S. B. Halstead, C. G. Hayes, A. Espinoza, V. Felices, R. Caceda, C T. Bautista, Y. Montoya, S. Douglas, and K. L Russell (2002). Effect of dengue-1 antibodies on American dengue-2 viral infection and dengue haemorrhagic fever. Lancet, 360(9329):310-312.

[46] C. Y. Kow, L. L. Koon, and P.F. Yin (2001). Detection of dengue viruses in field caught male Aedes aegypti and Aedes albopictus (Diptera: Culicidae) in Singapore by type-specific PCR. J Med Entomol, 38(4):475-479.

[47] Y. Kwok (September 24, 2010). Dengue Fever Cases Reach Record Highs, in Time. Retrieved August 4, 2014, from http://content.time.com/time/world/article/0, 8599, 2021265,00.html

[48] K. C. Leitmeyer, D. W. Vaughn, D. M. Watts, R. Salas, I. Villalobos, C. Ramos, and R. Rico-Hesse (1999). Dengue virus structural differences that correlate with pathogenesis. J Virol, 73(6):4738-4747.

[49] M. A. Lorono-Pino, C. B. Cropp, J. A. Farfan, A. V. Vorndam, E. M. Rodriguez-Angulo, E. P. Rosado-Paredes, L. F. Flores-Flores, B. J. Beaty, and D. J. Gubler (1999). Common occurrence of concurrent infections by multiple dengue virus serotypes. Am J Trop Med, 61(5):725-730.

[50] M. A. Loroño-Pino, J. A. Farfán-Ale, A. L. Zapata-Peraza, E. P. Rosado-Paredes, L. F. Flores-Flores, J. E. García-Rejón, F. J. Díaz, B. J. Blitvich, M. Andrade-Narváez, E. Jiménez-Ríos, C.D. Blair, K. E. Olson, W. Black IV, and B. J. Beaty (2004). Introduction of the american/asian genotype of dengue 2 virus into the Yucatan state of Mexico. Am J Trop Med, 71(4):485-492.

[51] E. Mamani, D. Figueroa, M. P. García, M. D. C. Garaycochea, and E. J. Pozo (2010). Concurrent infections by two dengue virus serotypes during an outbreak in northwestern peru, 2008. Revista Peruana de Medicina Experimental y Salud Pública, 27(1):16-21. 
[52] V.E.P. Martins, C. H. Alencar, M. T. Kamimura, F.M. de Carvalho Araujo, S. G. De Simone, R.F. Dutra, and M.I.F. Guedes (2012). Occurrence of natural vertical transmission of dengue-2 and dengue-3 viruses in Aedes aegypti and Aedes albopictus in Fortaleza, Ceara, Brazil. PloS one, 7(7):e41386.

[53] F. E. McKenzie and E. M. Samba (2004). The role of mathematical modeling in evidence-based malaria control. Am J Trop Med, 71(2 Suppl):94-96.

[54] J. A. Méndez, J. A. Usme-Ciro, C. Domingo, G. J. Rey, J. A. Sánchez, A. Tenorio, and J. C. Gallego-Gomez (2012). Phylogenetic reconstruction of dengue virus type 2 in Colombia. Virology, 9:64.

[55] D. Mir, H. Romero, L. M. F. de Carvalho, and G. Bello (2014). Spatiotemporal dynamics of DENV-2 Asian-American genotype lineages in the Americas. PloS one, 9(6):e98519.

[56] Y. Montoya, S. Holechek, O. Caceres, A. Palacios, J. Burans, C. Guevara, F. Quintana, V. Herrera, E. Pozo, E. Anaya, E. Mamani, V. Gutierrez, A. Ladron de Guevara, E. Fernandez, P. Asmat, V. Alva-Davalos, C. Hoguin, V. A. Laguna, A. M. Morales, P. Minaya, and T. Kochel (2003). Circulation of dengue viruses in North-Western Peru, 2000-2001. Dengue Bulletin, 27:52-62.

[57] D. M. Morens and A.S. Fauci (2008). Dengue and hemorrhagic fever: A potential threat to public health in the United States. JAMA, 299(2):214-216.

[58] J. Mota and R. Rico-Hesse (2009). Humanized mice show clinical signs of dengue fever according to infecting virus genotype. $J$ Virol, 83(17):8638-8645.

[59] J. D. Murray (2002). Mathematical Biology I: An Introduction, vol. 17 of Interdisciplinary Applied Mathematics. Springer.

[60] Nguyen T.H., Nguyen. T. L., H.-Y. Lei, Y.-S. Lin, Le B.L., K.-J. Huang, C.-F. Lin, Do Q.H., Vu T. Q. H., Lam T. M., T.-M. Yeh, J.-H. Huang, C.-C. Liu, S. B. Halstead (2006). Volume replacement in infants with dengue hemorrhagic fever/dengue shock syndrome. Am J Trop Med, 74(4):684-691.

[61] H. Nishiura (2006). Mathematical and statistical analyses of the spread of dengue. Dengue Bulletin, 30:51.

[62] D. Normile (2013). First New Dengue Virus Type in 50 Years. Science, 342(6157):415.

[63] R. Rico-Hesse, L. M. Harrison, R. A. Salas, D. Tovar, A. Nisalak, C. Ramos, J. Boshell, M. T. R. de Mesa, R. MR. Nogueira, and A. T. Rosa (1997). Origins of dengue type 2 viruses associated with increased pathogenicity in the Americas. Virology, 230(2):244251.

[64] F. Rodhain and L. Rosen (1997). Mosquito vectors and dengue virus-vector relationships. Dengue and dengue hemorrhagic fever, 45-60.

[65] L. Rosen, D. A. Shroyer, R. B. Tesh, J. E. Freier, and J. C. Lien (1983). Transovarial transmission of dengue viruses by mosquitoes: Aedes albopictus and Aedes aegypti. Am J Trop Med, 32(5):1108-1119.

[66] R. Ross (1910). The prevention of malaria. Dutton.

[67] F. Sanchez, D. Murillo, and C. Castillo-Chavez (2012). Change in Host Behavior and its Impact on the Transmission Dynamics of Dengue. BIOMAT 2011 International Symposium on Mathematical and Computational Biology. Edited by Rubem P. Mondaini, 9(10):1-13. 
[68] N. Sangkawibha, S. Rojanasuphot, S. Ahandrik, S. Viriyapongse, S. Jatanasen, V. Salitul, B. Phanthumachinda, and S. B. Halstead (1984). Risk factors in dengue shock syndrome: a prospective epidemiologic study in Rayong, Thailand: I. The 1980 outbreak. Am J Epidemiol, 120(5):653-669.

[69] T. Sasaki, C. Setthapramote, T. Kurosu, M. Nishimura, A. Asai, M. D. Omokoko, C. Pipattanaboon, P. Pitaksajjakul, K. Limkittikul, A. Subchareon, P. Chaichana, T. Okabayashi, I. Hirai, P. Leaungwutiwong, R. Misaki, K. Fujiyama, K. Ono, Y. Okuno, P. Ramasoota, and K. Ikuta (2013). Dengue virus neutralization and antibodydependent enhancement activities of human monoclonal antibodies derived from dengue patients at acute phase of secondary infection. Antiviral research, 98(3):423-431, 2013.

[70] E. Shim, Z. Feng, and C. Castillo-Chavez (2012). Differential impact of sickle cell trait on symptomatic and asymptomatic malaria. Math Biosci Eng, 9(4):877.

[71] J.F. Siler, M. W. Hall, and A.P. Hitchens (1926). Dengue: its history, epidemiology, mechanism of transmission, etiology, clinical manifestations, immunity, and prevention. Philippine Bur. Sci., (Monogr.20).

[72] J. S. Simmons, J.H. St John, and F.H. Reynolds (1931). Experimental studies of dengue. Mongr. Bur. Sci. Philipp. Is., (29).

[73] N. Soderlund, K. Zwi, A. Kinghorn, and G. Gray (1999). Prevention of vertical transmission of HIV: analysis of cost effectiveness of options available in South Africa. $B m j$, 318(7199):1650-1656.

[74] S. C. Tewari, V. Thenmozhi, C. R. Katholi, R. Manavalan, A. Munirathinam, and A. Gajanana (2004). Dengue vector prevalence and virus infection in a rural area in south India. Trop Med Int Health, 9(4):499-507.

[75] P. Van den Driessche and J. Watmough (2002). Reproduction numbers and subthreshold endemic equilibria for compartmental models of disease transmission. Math Biosci, 180(1):29-48.

[76] A. P. Vilela, L. B. Figueiredo, J. R. dos Santos, Á. E. Eiras, C. A. Bonjardim, P. C. Ferreira, and E. G. Kroon (2010). Dengue virus 3 genotype I in Aedes aegypti mosquitoes and eggs, Brazil, 2005-2006. Emerg Infect Dis, 16(6):989.

[77] D. M. Watts, B. A. Harrison, S. Pantuwatana, T. A. Klein, and D. S. Burke (1985). Failure to detect natural transovarial transmission of dengue viruses by aedes aegypti and aedes albopictus (diptera: Culicidae). J Med Entomol, 22(3):261-265.

[78] D. M. Watts, K. R. Porter, P. Putvatana, B. Vasquez, C. Calampa, C. G. Hayes, and S. B. Halstead (1999). Failure of secondary infection with american genotype dengue 2 to cause dengue haemorrhagic fever. Lancet, 354(9188):1431-1434.

[79] World Health Organization. (2012). Dengue and severe dengue fact sheet. World Health Organization.

[80] P. Zhang, G. J. Sandland, Z. Feng, D. Xu, and D. J. Minchella (2007). Evolutionary implications for interactions between multiple strains of host and parasite. J Theor Biol, 248(2): 225-240. 